\title{
Deficient HER3 expression in poorly-differentiated colorectal cancer cells enhances gefitinib sensitivity
}

\author{
SUSUMU NAKATA ${ }^{1}$, HARUNARI TANAKA ${ }^{1}$, YUICHI ITO ${ }^{2}$, MASAYASU HARA ${ }^{3}$, MITSUGU FUJTA ${ }^{4}$, \\ EISAKU KONDO $^{1}$, YUKIHIDE KANEMITSU ${ }^{5}$, YASUSHI YATABE ${ }^{6}$ and HAYAO NAKANISHI ${ }^{7}$
}

\author{
${ }^{1}$ Division of Oncological Pathology, Aichi Cancer Center Research Institute; ${ }^{2}$ Department of Gastroenterological Surgery, \\ Aichi Cancer Center Central Hospital; ${ }^{3}$ Department of Gastroenterological Surgery, Nagoya City University, \\ Nagoya 464-8681; ${ }^{4}$ Department of Microbiology, Kinki University, Graduate School of Medical Sciences, \\ Osaka 589-8511; ${ }^{5}$ Colorectal Surgery Division, National Cancer Center Hospital, Tokyo 104-0045; \\ ${ }^{6}$ Department of Pathology and Molecular Medicine, Aichi Cancer Center Central Hospital, Nagoya 464-8681; \\ ${ }^{7}$ Laboratory of Pathology and Clinical Research, Aichi Cancer Center Aichi Hospital, Okazaki 444-0011, Japan
}

Received April 16, 2014; Accepted May 30, 2014

DOI: 10.3892/ijo.2014.2538

\begin{abstract}
Poorly-differentiated colorectal cancers (PD-CRC) show high metastatic potential and poor prognosis. However, molecular characteristics of PD-CRC remain unknown to date, particularly in molecular targeting therapy for patients with PD-CRC. In this study, we examined the expression of EGFR, HER2 and HER3 in PD-CRC by immunohistochemical analysis of archived clinical specimens of primary tumors and investigated the sensitivity of PD-CRC cell lines to gefitinib, a tyrosine kinase inhibitor for EGFR in vitro. We found that HER3 expression of PD-CRC among members of the HER family was significantly lower than that of well to moderately differentiated CRC (WMD-CRC) and 37\% of the PD cases showed a EGFR ${ }^{+} / \mathrm{HER}^{+} / \mathrm{HER}^{-}$- expression pattern. COLM-5 cells, a PD-CRC-derived cell line, which exhibits $\mathrm{EGFR}^{+} / \mathrm{HER}^{+} / \mathrm{HER}^{-}$expression pattern and recapitulates the typical histology of PD-CRC in xenografted tumors, showed high gefitinib sensitivity both in vitro and in vivo, compared with WMD-CRC cell line (COLM-2). Treatment with gefitinib resulted in the upregulation of $\mathrm{p} 27^{\mathrm{Kip} 1}$ expression and induction of G1 cell cycle arrest, concomitantly associated with inactivation of PI3K/Akt signaling in COLM-5 cells and marked inhibition of xenografted tumors in nude mice, but not evident in COLM-2 cells. Treatment with sodium butyrate, an HDAC inhibitor that induces differentiation, upregulated the expression of HER3 associated with enhancement of the PI3K/Akt signaling, attenuated gefitinib-mediated p2 $7^{\mathrm{Kip} 1}$
\end{abstract}

Correspondence to: Dr Hayao Nakanishi, Laboratory of Pathology and Clinical Research, Aichi Cancer Center Aichi Hospital, 18 Kuriyado, Ketsu-machi, Okazaki 444-0011, Japan

E-mail: hnakanis@aichi-cc.jp

Key words: poorly-differentiated colorectal cancer, HER3, EGFR, gefitinib, p27 upregulation and reduced gefitinib sensitivity in COLM-5 cells in vitro. Furthermore, enforced expression of HER3 in COLM-5 cells resulted in significant resistance to gefitinib treatment both in vitro and in vivo. These findings suggest that deficient HER3 expression plays an important role in gefitinib sensitivity and that a malignant subset of $\mathrm{PD}$ with $\mathrm{EGFR}^{+} /$ HER $2^{+} /$HER $^{-}$- phenotype is a potential candidate for a target of anti-EGFR molecular therapy such as gefitinib.

\section{Introduction}

The majority of CRC are well- to moderately-differentiated adenocarcinomas (WMD-CRC) with apparent glandular morphology. Pathogenesis of such a differentiated-type CRC is a multistep process, recognized as an adenoma-carcinoma sequence, starting from a polypoid lesion such as adenoma, with subsequent progression to adenocarcinoma, and finally metastatic adenocarcinoma (1). Throughout this process, a series of genetic alterations such as mutation in $A P C, \beta$-catenin, $K R A S, p 53$ and $T G F-\beta R-I I$ and microsatellite instability (MSI) are known to be accumulated with disease progression (2). Among these changes, mutations in Wnt signaling cascade such as $A P C$ and $\beta$-catenin occur at the initial step and play a key role in carcinogenesis of WMD-CRC (3). In contrast, poorly-differentiated adenocarcinomas (PD-CRC), defined by minimal glandular structure (1), are relatively rare accounting for only $5-10 \%$ of CRCs (4), and molecular pathogenesis and clinicopathological features are reportedly distinct from WMD-CRC (5). PD-CRC is heterogeneous and has been subclassified into several types (6). One relatively rare subset of PD-CRC is 'medullary type adenocarcinomas' which are characterized by MSI-positive, right-sided, scant fibrous stroma and relatively favorable prognosis (7). However, most PD-CRCs are aggressive and highly metastatic, with a less favorable prognosis than that of WMD-CRC (8-10). As for genetic alterations, several investigators reported that PD showed a lower incidence of KRAS mutation, a higher incidence of $B R A F$ mutation, MSI, a higher promoter methylation 
of P16 than WMD-CRC (11-13). However, the molecular pathogenesis and therapeutic target specific for $\mathrm{PD}$ still remain largely unknown.

The aberrantly activated HER family of receptor tyrosine kinases including EGFR, HER2, HER3 and HER4 are associated with carcinogenesis and tumor progression, and therefore are major molecular targets in various epithelial malignancies including gastrointestinal cancers $(14,15)$. Among them, EGFR overexpression such as gene amplification was reported in $12-17 \%$ of CRC patients and activation of EGFR results in acceleration of growth and survival of tumor cells through the MAPK or PI3K/Akt pathways and correlates with a poor patient outcome (16). Molecular targeting therapy with monoclonal antibody to EGFR (cetuximab) has been clinically used in patients with metastatic CRC in combination with chemotherapy (17). However, EGFR expression as assessed by immunohistochemistry proved to be insufficient to predict cetuximab sensitivity (18). Alternatively, KRAS mutation at codons 12 or 13 , and more recently $B R A F$ mutation (V600E) have been reported as promising new predictive markers for cetuximab-resistance in patients with metastatic CRC (19-21).

Unlike EGFR, HER3 has a unique kinase-inactive nature because of the amino acid substitutions in critical residue in the tyrosine kinase domain, and is therefore not a direct target for HER tyrosine kinase inhibitor. Upon binding with heregulin ligand, HER3 can be transphosphorylated and then successfully activate PI3K, via its multiple docking sites for the p85 regulatory subunit of PI3K and dimerization with other HER members such as HER2 (22). Therefore, HER3 plays a key regulatory role in transducing signals downstream to PI3K/Akt pathways. Recent studies demonstrated that HER3 overexpression and consequent activation of PI3K/Akt signaling lead to resistance to tyrosine kinase inhibitors such as gefitinib in HER 2 overexpressing breast cancer, because of reactivation of HER3 signaling after transient inhibition by the drugs and the difficulty of consistent blocking HER3/PI3K/Akt pathway (23). HER3/PI3K/Akt pathway also plays a negative regulatory role in $\mathrm{p} 27^{\mathrm{Kip} 1}$-mediated $\mathrm{G} 1$ cell cycle arrest by trastuzumab in breast cancers (24). Furthermore, HER3 is frequently overexpressed in CRC (25). These findings suggest a potential role of HER3 in responsiveness of CRC to EGFR targeting therapy and that HER3 is therefore a potential predictive marker for drug resistance or susceptibility. To date, however, the functional and clinical significance of HER3 expression in PD-CRC is not entirely known.

In the present study, we found that HER3 expression in a clinical specimen of primary PD-CRC is significantly lower compared with that of WMD-CRC. Furthermore, we investigated the molecular mechanism of gefitinib sensitivity using a HER3-deficient and metastatic PD-CRC cell line that exhibits high sensitivity to gefitinib. Our findings raise the possibility of EGFR-targeting therapeutics as a new strategy against highly malignant PD-CRC.

\section{Materials and methods}

Compounds. Gefitinib (ZD1839; Iressa) was provided by AstraZeneca (Macclesfield, UK). Human recombinant heregulin was obtained from R\&D Systems (Minneapolis,
$\mathrm{MN})$. For western blot analysis, rabbit polyclonal anti-Akt, phospho-Akt (Ser473) (Cell Signaling, Beverly, MA), PI3Kp85 (Upstate, Lake Placid, NY), mouse monoclonal anti-EGFR, HER2, p21 ${ }^{\text {WAF1/CIP }}$ (all from Cell Signaling), HER3 (clone 2F12, Upstate), p27 ${ }^{\mathrm{Kip} 1}$ (BD Pharmingen, San Diego, CA), Skp2 (Zymed, Carlsbad, CA), $\beta$-actin (BD Pharmingen) and rabbit monoclonal anti-phospho-HER3 (Tyr1289) (Cell Signaling) were used. For immunohistochemistry, mouse monoclonal anti-E cadherin, vimentin (Dako, Glostrup, Denmark), $\beta$-catenin (BD Pharmingen), EGFR (Novocastra, Newcastle, UK), rabbit polyclonal anti-HER2 (Dako), p2 $7^{\text {Kipl }}$ (Thermo Scientific, Fremont, CA), rabbit monoclonal anti-HER3 (D22C5, Cell Signaling) were used. For flow cytometry, mouse monoclonal anti-EGFR (NeoMarkers), HER2 (44E7, Cell Signaling), and HER3 (Ab-10, NeoMarkers) were used.

Patients. A total of 62 colorectal cancer patients consisting of 38 WMD-CRC and 24 PD-CRC patients were enrolled in this study. PD-CRC (1998-2002) and WMD-CRC patients (1998) underwent operation at the Department of Gastroenterological Surgery, Aichi Cancer Center Central Hospital. Analysis using these clinical samples was carried out under approval by the institutional ethics review board of Aichi Cancer Center.

Immunohistochemical analysis of primary colorectal cancers. Surgically resected specimens were fixed in $10 \%$ buffered formalin and embedded in paraffin. For antigen retrieval, the sections were treated with microwave at $98^{\circ} \mathrm{C}$ for $10 \mathrm{~min}$ in citrate buffer $\mathrm{pH}$ 6.0. After blocking of nonspecific reactions by normal serum for $30 \mathrm{~min}$, these sections were incubated at $4^{\circ} \mathrm{C}$ overnight with primary antibodies, thoroughly washed in PBS, then incubated with biotinylated secondary antibodies. The sections were washed again with PBS and incubated with streptavidin-peroxidase complex (Vectastain ABC kit, Vector Laboratories, Burlingame, CA) for $60 \mathrm{~min}$. The sites of peroxidase binding were visualized using $0.01 \%$ diaminobenzidine (DAB) as a chromogen.

Expression of EGFR and HER2 protein was scored for each of the $3+, 2+, 1+$ and 0 categories based on the membrane staining according to the criteria of HercepTest. In this study, we subclassified cases into 3 classes as negative, 0 ; weakly positive staining, 1+; strongly positive staining, 2+ and 3+. For HER3, the staining pattern of the CRC was both membranous and cytoplasmic, and the latter cytoplasmic pattern was more prominent compared with that of EGFR/HER2, but we evaluated cases based on the membranous staining as negative, 0 ; weakly positive staining, 1+; strongly positive staining, $2+$ and $3+$, like EGFR/HER2.

Cell lines. COLM-5 cell line was established from liver metastasis of PD-CRC Japanese patient, and COLM-2 was established from WMD-CRC Japanese patient as described previously (26). These cell lines were cultured in Dulbecco's modified Eagle's medium (DMEM; Nissui Pharmaceutical Co., Tokyo, Japan) supplemented with $10 \%$ fetal bovine serum (FBS) (Gibco, Grand Island, NY), $100 \mathrm{U} / \mathrm{ml}$ penicillin and $100 \mu \mathrm{g} / \mathrm{ml}$ streptomycin (Falcon, BD Labware, Franklin Lakes, NJ) in a humidified $5 \% \mathrm{CO}_{2}$ incubator at $37^{\circ} \mathrm{C}$. To evaluate metastasis clearly with green fluorescence, these cells were transfected with the pEGFP-C1 plasmid (Clontech Laboratories, Palo Alto, 
CA) using FuGENE6 transfection reagent (Roche Diagnostics, Basel, Switzerland).

In vitro growth inhibition assay. The cells were plated at a density of $1 \times 10^{4}$ cells/well in 96-well plastic plates (Falcon) and treated with increasing doses of gefitinib $(0.1,1.0$ and $10 \mu \mathrm{M})$. The number of viable cells was assessed with a trypan-blue dye exclusion test on day 3 by counting using a hemocytometer in quadruplicate.

Measurement of caspase-3/7 activity. Cells were plated at $1 \times 10^{4}$ cells in 96-well plates, and cultured in the presence of gefitinib $(0.1,1.0$ and $10 \mu \mathrm{M})$ for $24 \mathrm{~h}$. Caspase-3/7 activity was then measured using Apo-One Homogenous caspase-3/7 Assay Kit (Promega Co., Madison, WI) according to the manufacturer's instructions.

FISH analysis. Amplification of the EGFR and HER2 gene was determined by dual-color FISH method using Passvision EGFR or HER2 DNA probe kit (Vysis Inc., Downers Grove, IL) according to the manufacturer's protocol. The nucleus was counterstained with 4',6-diamidino-2-phenylindole (DAPI). The slides were observed under BX60 fluorescence microscope equipped with a DP50 digital camera (Olympus, Tokyo, Japan). A cell was considered to have amplification when a signal ratio of specific probe to the centromere region of the corresponding chromosome was more than 2 .

Flow cytometry. Cells were suspended in PBS and then incubated with mouse monoclonal antibodies to human EGFR, HER2 and HER3 for 30 min on ice. After washing with PBS containing $0.5 \%$ bovine serum albumin (BSA) and $5 \mathrm{mM}$ EDTA, they were exposed to FITC (PE)-conjugated anti-mouse IgG (Molecular Probes) for $30 \mathrm{~min}$. The intensity of fluorescence was measured by FACSCalibur (BD Biosciences, San Diego, CA).

Cell cycle analysis. Cells were treated with gefitinib at a concentration of $10 \mu \mathrm{M}$ for $24 \mathrm{~h}$, and stained with propidium iodide using CycleTest Plus Kit (Becton Dickinson, San Jose, CA) according to the manufacturer's instructions. Flow cytometric analysis was done in FACSCalibur (BD Biosciences). Data collected from 10,000 cells for each experiment were analyzed by ModFit software (Verity Software House, Topsham, ME).

Western blot analysis. To evaluate the effects of gefitinib under ligand-mediated stimulation, cells were incubated in serum-free medium for $24 \mathrm{~h}$, and exposed to gefitinib for $2 \mathrm{~h}$ at $37^{\circ} \mathrm{C}$. Cells were then incubated for 5-10 min with heregulin $(10 \mathrm{ng} / \mathrm{ml})$. Cells were then lysed at $4^{\circ} \mathrm{C}$ in lysis buffer $[25 \mathrm{mM}$ Tris-HCl, pH 7.5, 150 mM NaCl, 0.1\% NP-40, 1 mM EDTA and protease inhibitor Cocktail plus phosphatase inhibitor Cocktail (Roche Diagnostics, Mannheim, Germany)]. A total of $10 \mu \mathrm{g}$ of the samples were separated by SDS-PAGE, transferred to Immune-Blot PVDF Membrane (Bio-Rad), immunoblotted with antibodies described above, and visualized using Super Signal West Pico (Dura) Chemiluminescence Substrate (Thermo Scientific).

RT-PCR assay. Total RNA was extracted using Isogen (Nippon Gene, Tokyo, Japan) and cDNA was then synthesized with
SuperScript II reverse transcriptase (Invitrogen, Carlsbad, CA) according to the manufacturer's protocol. A reverse transcription-polymerase chain reaction assay (RT-PCR) for $\mathrm{p} 27^{\mathrm{Kip} 1}$ and HER3 was performed using specific oligonucleotide primer pairs and probes \#60, \#37, respectively (Universal Probe Library, Roche Diagnostics), on the LightCycler instrument (Roche Diagnostics). As an internal control, glyceraldehyde3-phosphate dehydrogenase (GAPDH) was used.

Xenograft studies for anti-tumor and anti-metastatic activity. All animal experiments were carried out under the approval of the Institutional Ethics Committee for Animal Experiment of Aichi Cancer Center Research Institute. Five millions cells were injected subcutaneously into the left abdominal flanks of 8-week-old male nude mice of KSN strain (Shizuoka Laboratory Animal Center, Hamamatsu, Japan). For gefitinib treatment, mice $(n=5)$ were orally administered gefitinib with a gastric tube at a dose of 0 or $150 \mathrm{mg} / \mathrm{kg} /$ day from $1-2$ weeks post-injection, five times per week for 3-4 weeks. In the control groups, mice were orally administered vehicle ( $0.5 \%$ polysorbate, Merck, Darmstadt, Germany). Tumor maximum diameter (L) and the right angle diameter to that axis (W) were measured with a slide caliper every week. Tumor volume was calculated by the following formula, $\mathrm{L} \times \mathrm{W} \times \mathrm{W} \times 1 / 2$. To assess lung metastasis, we pretreated nude mice 4 times with intraperitoneal injection of rabbit antiserum to bovine asialo GM1 (400 $\mu \mathrm{g} / 0.2 \mathrm{ml} / \mathrm{mouse})$ (Wako Pure Chemical, Osaka, Japan) before (once) and after (3 times) transplantation, which resulted in a substantial increase in lung metastasis via depletion of NK cells. A million of GFP-tagged cells were then injected intravenously, and macroscopic lung metastases were evaluated by counting visible parietal nodules in mice with or without gefitinib treatment at 3 weeks after injection. For peritoneal metastasis, $3 \times 10^{6}$ cells were injected intraperitoneally. At 4 weeks post-injection, mice with or without gefitinib treatment were sacrificed, and peritoneal metastases were detected macroscopically and quantified as described previously (27).

Overexpression of HER3 in COLM-5 cells. A cDNA of HER3 open reading frame was purchased from Origene (Rockville, MD), and inserted into the pcDNA3.2-DEST vector (Invitrogen). The HER3 cDNA was confirmed by sequencing. The expression vector was introduced in COLM-5 cells by FuGENE-6 reagent according to manufacturer's instruction. Stable HER3 expressing COLM-5 cells were established by a selection with $\mathrm{G} 418$ reagent.

Statistical analysis. The statistical significance of differences in growth was analyzed using the Student's t-test. Differences in the incidence between groups were analyzed with Fisher's exact test. A p-value $<0.05$ was considered significant.

\section{Results}

Deficient or low HER3 expression in the primary PD-CRC. We examined EGFR, HER2 and HER3 expression of 24 PD-CRC and 38 WMD-CRC by an immunohistochemical analysis (Fig. 1A). EGFR positivity rate (weakly positive and strongly positive) of PD-CRC (71\%) was almost comparable 
A
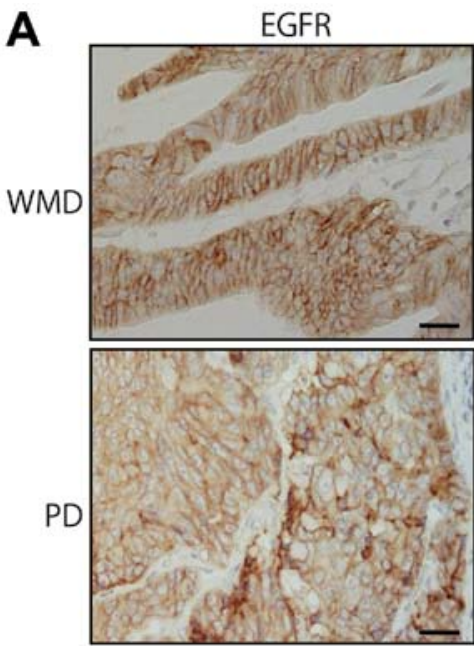

HER2
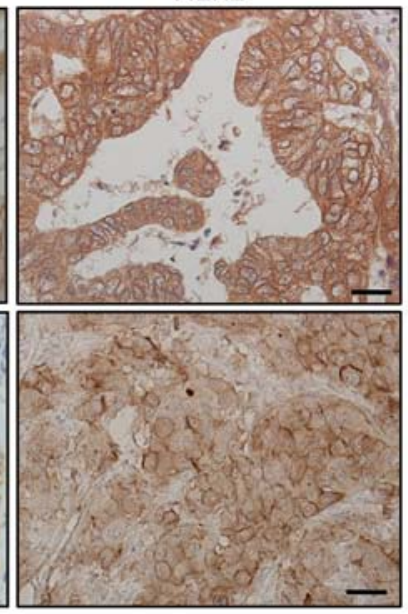

HER3
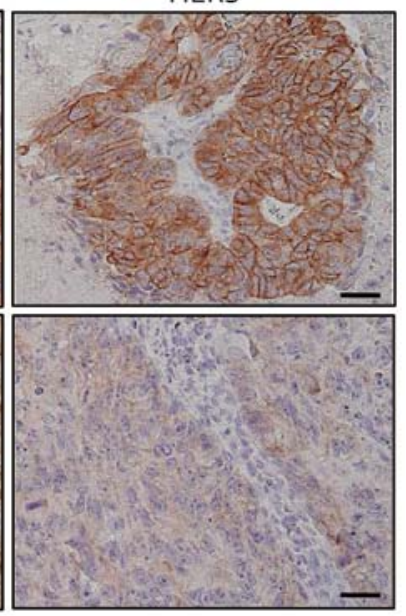

B

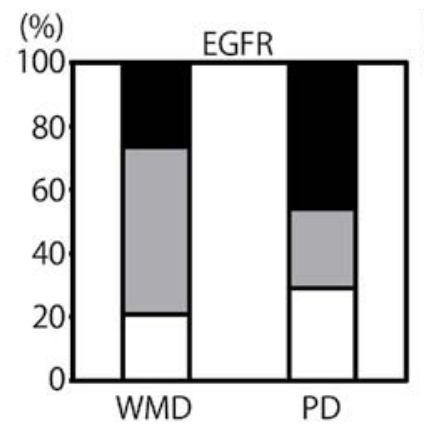

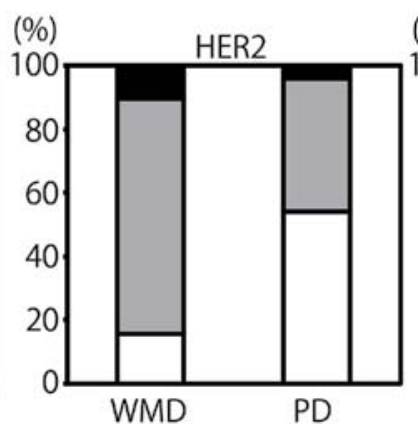

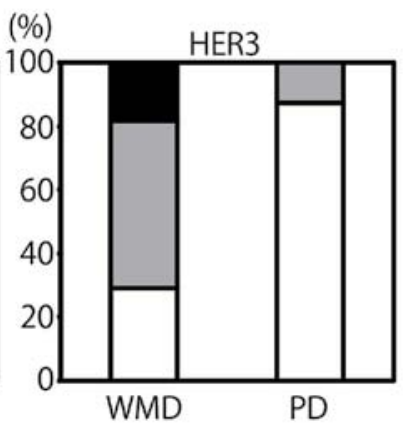

Figure 1. Comparison of EGFR, HER2 and HER3 expression between WMD-CRC and PD-CRC tumors by immunohistochemistry. (A) Representative expression patterns of HER family in WMD-CRC and PD-CRC. Bars, $50 \mu \mathrm{m}$. (B) Comparison of the incidence of positive staining for EGFR, HER2 and HER3 between WMD-CRC and PD-CRC. Strongly positive staining (black bars), weakly positive staining (grey bars) and negative staining (white bars).

to that of WMD-CRC (79\%), but for the EGFR strong membranous staining, the positivity rate of PD $(46 \%)$ was higher than WMD-CRC (26\%) (Fig. 1B). HER2 positivity rate is higher in WMD-CRC $(84 \%)$ than PD-CRC $(46 \%)$. However, circumferential strong membranous staining $(2+$ and 3+) like EGFR was relatively rare even in WMD-CRC (11\%). HER3 expression localized both in the cytoplasm and the membrane and the positivity rate was significantly lower in PD-CRC (12\%) than WMD-CRC (71\%), indicating that the majority of PD-CRC is HER3-negative. HER3 positivity rate was significantly different between WMD-CRC and PD-CRC ( $<<0.01$, Fisher's exact test) (Fig. 1A and B). Tumors with EGFR-positive, HER2-positve and HER3-negative phenotype, designated by EGFR ${ }^{+} / \mathrm{HER}^{+} / \mathrm{HER}^{-}$, accounted for $37 \%$ of total PD cancers.

COLM-5 PD-CRC cells express EGFR and HER2, but not HER3. COLM-5 cells established from a resected liver metastasis of a PD-CRC patient formed subcutaneous tumors with histological features of poorly-differentiated adenocarcinoma in nude mice (Fig. 2A). COLM-5 tumors were strongly stained for vimentin and slug, but still maintained epithelial characteristics such as scant cytokeratin and E-cadherin staining, indicating their feature of epithelialmesenchymal transition (EMT). COLM-5 tumors exhibited nuclear staining of P53 (data not shown), but not $\beta$-catenin
(Fig. 2A) and no KRAS and $B R A F$ mutation was observed (data not shown). In contrast, COLM-2 xenografts exhibited typical features of moderately-differentiated adenocarcinoma with glandular formation, which were strongly positive for cytokeratin and E-cadherin. The COLM-2 cells showed nuclear accumulation of $\beta$-catenin like the majority of WMD-CRC (data not shown).

Immunohistochemical analysis of transplanted tumors in nude mice showed that the COLM-5 cells were strongly positive (2+ and 3+) for both EGFR and HER2, respectively, based on the membrane staining, but negative for HER3 on their surface. However, FISH analysis demonstrated neither EGFR nor HER2 gene amplification in COLM-5 cells (Fig. 2B). Flow cytometric analysis confirmed that COLM-5 cells were deficient of HER3 expression on their surface (Fig. 2C). Western blot analyses of the cultured cells further confirmed overexpression of EGFR and HER2 and deficient HER3 expression of COLM-5 cells, whereas COLM-2 cells overexpressed EGFR, HER2 and HER3 (Fig. 2D).

COLM-5 cells exhibit high sensitivity to gefitinib. Gefitinib significantly inhibited growth of COLM-5 cells in vitro more strongly than COLM-2 cells $(\mathrm{p}<0.05)$ (Fig. 3A). Cell cycle analysis by flow cytometry revealed that gefitinib treatment decreased $S$ phase cells from 27.3 to $14.0 \%$ and increased G0-G1 phase cells from 63.4 to $80.9 \%$ in COLM-5 cells, 
A

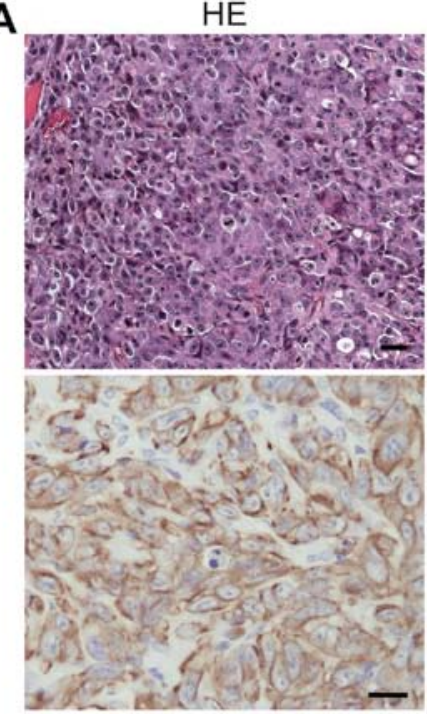

Vimentin

B

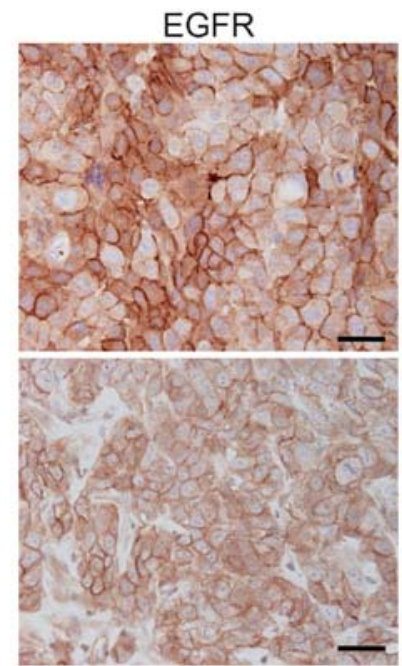

HER2

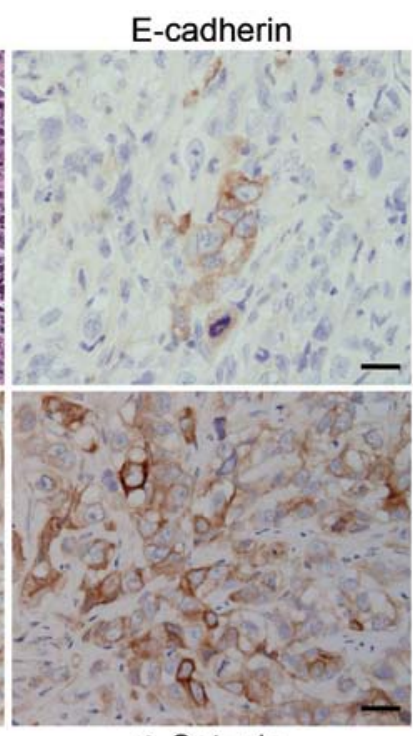

$\beta$-Catenin
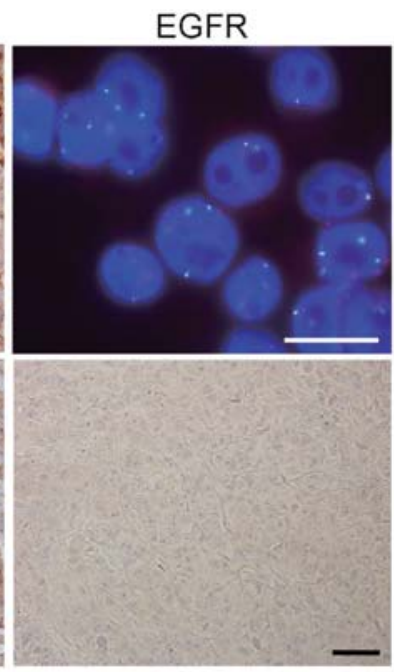

HER3
C
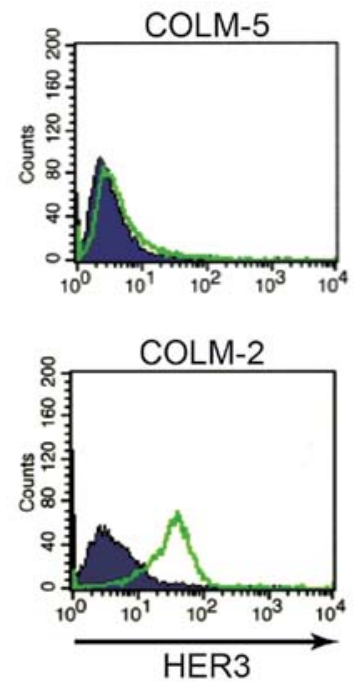

D

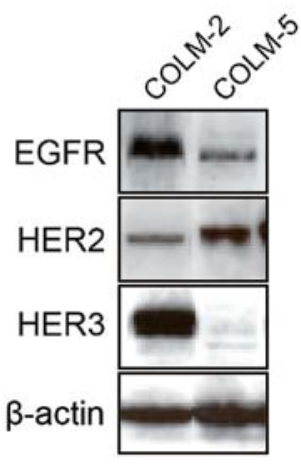

Figure 2. Biological characteristics and HER family expression of COLM-5 cell line. (A) Histological analysis of subcutaneous (sc) tumors in nude mice showing poorly-differentiated carcinomatous appearance without glandular structures (HE). Immunohistochemical staining of E-cadherin, vimentin and $\beta$-catenin in sc tumors. Bars, $50 \mu \mathrm{m}$. (B) EGFR, HER2 and HER3 expression of COLM-5 xenografts (bars, $50 \mu \mathrm{m}$ ) and FISH analysis of the EGFR gene locus (upper right). (C) Flow cytometric analysis of HER3 expression in COLM-5 and COLM-2 cells. (D) EGFR, HER2 and HER3 protein expression of cultured COLM-5 and COLM-2 cells as determined by western blot analysis.

indicating significant induction of G1 arrest of cell cycle (Fig. 3B). In contrast, no G1 cell cycle arrest by gefitinib in COLM-2 cells was observed (data not shown). Caspase-3/7 assay showed that significant apoptosis was not induced by gefitinib in vitro in COLM-5 or COLM-2 cells even at high concentrations (Fig. 3C).

Gefitinib upregulates $p 27^{\text {Kipl }}$ and attenuates the HER3/PI3K/Akt signaling pathway in COLM-5 cells. To gain insights into molecular mechanisms underlying the sensitivity to gefitinib of COLM-5 cells, we examined the expression of $\mathrm{p} 27^{\mathrm{Kip} 1}$ and $\mathrm{p} 21^{\mathrm{WAF} / \mathrm{CIP} 1}$. In COLM-5 cells, $\mathrm{p} 27^{\mathrm{Kipl}}$ protein was weakly expressed under basal condition, and significantly increased by gefitinib treatment in a dose-dependent manner (Fig. 4A). We confirmed that gefitinib treatment upregulates p2 $7^{\text {Kipl }}$ also at mRNA level by RT-PCR analysis (Fig. 4B). In contrast, COLM-2 cells highly expressed both $\mathrm{p} 27^{\mathrm{Kip} 1}$ and $\mathrm{p} 21^{\mathrm{WAF} 1 / \mathrm{CIP} 1}$, but the expression was unchanged after gefitinib treatment (Fig. 4A). No p21 ${ }^{\mathrm{WAF} / \mathrm{CIP} 1}$ expression was detected in COLM-5 cells. Expression level of Skp2, which regulates p2 $7^{\mathrm{Kip} 1}$ expression at protein level $(28,29)$, did not change by gefitinib treatment (Fig. 4A), suggesting that $27^{\text {Kipl }}$ was induced at a transcriptional level. An immunofluorescence analysis confirmed nuclear relocalization of $\mathrm{p} 27^{\mathrm{Kip} 1}$ from cytoplasm after gefitinib treatment (Fig. 4C). Upon stimulation with heregulin, increase in phosphorylation of HER3 and Akt was detectable in COLM-5 cells, but much weaker than in COLM-2 cells. The slight increase in phophorylation of Akt by heregulin was almost completely reversed by gefitinib in COLM-5 cells, whereas such a suppression of phophorylation of Akt by gefitinib was slight in COLM-2 cells (Fig. 4D). 


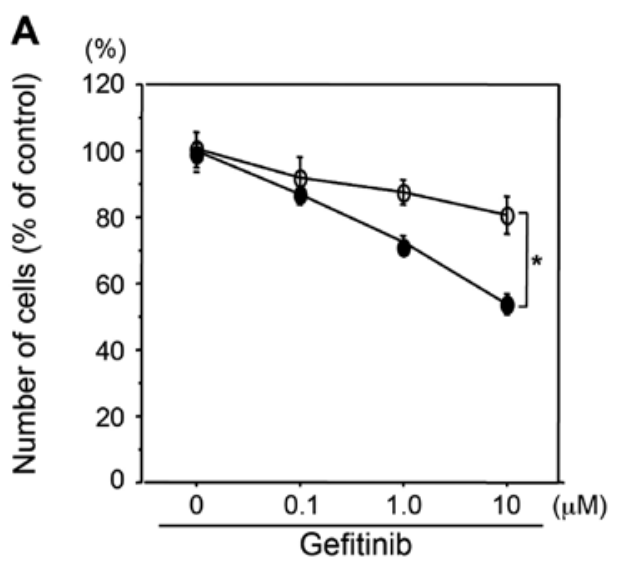

B
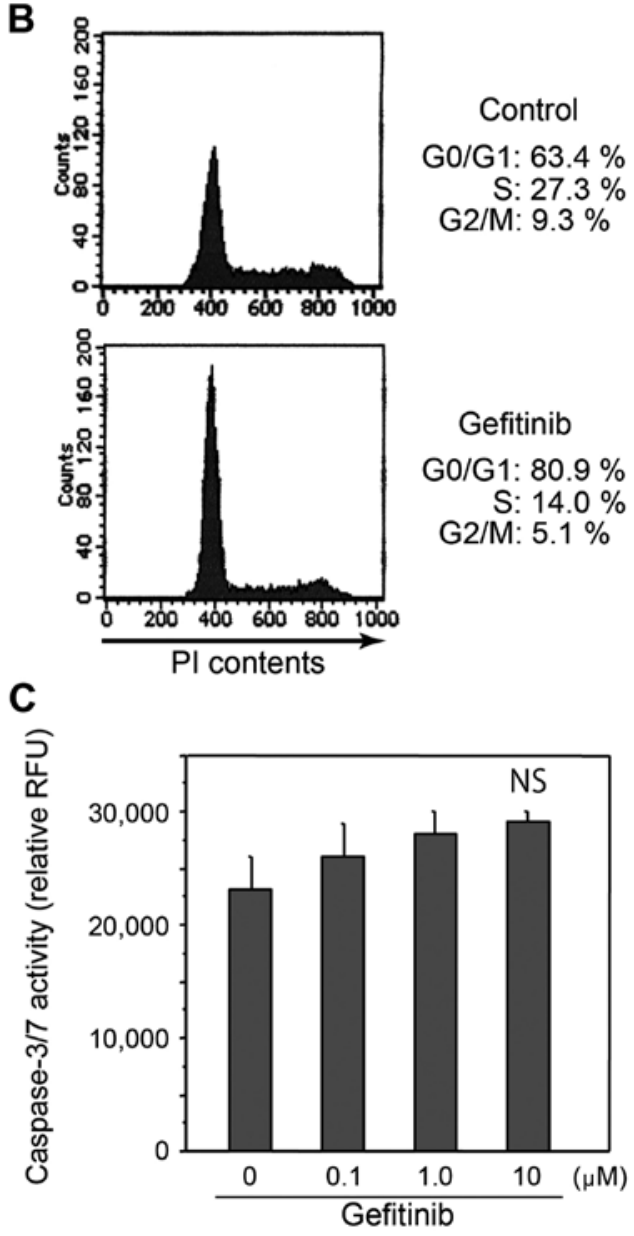

Figure 3. Effects of gefitinib on the growth, apoptosis and cell cycle of COLM-5 and COLM-2 cell lines. (A) Inhibition of growth by gefitinib in COLM-2 (O) and COLM-5 (•) cells in vitro. "p<0.05. (B) Cell cycle analysis of COLM-5 cells with or without gefitinib treatment by flow cytometry. (C) Apoptosis induction by gefitinib in COLM- 5 cells as evaluated by measuring caspase $3 / 7$ activity. Bars represent mean \pm SE. NS, not significant.

Gefitinib inhibits COLM-5 xenografts and metastases in vivo. Antitumor effect of gefitinib in vivo was examined using subcutaneous (sc) transplanted tumors in nude mice. Treatment with gefitinib markedly suppressed sc tumor growth of COLM-5 xenografts $(\mathrm{p}<0.01)$, but did not significantly suppress COLM-2 xenografts (Fig. 5A). BrdU incorporation was significantly reduced in gefitinib-treated COLM-5 tumors (Fig. 5B). Furthermore, increase in nuclear accumulation of $\mathrm{p} 27^{\mathrm{Kip} 1}$ protein after gefitinib treatment for 3 weeks was observed in COLM-5 tumors (Fig. 5B). Such remarkable antitumor effects of gefitinib was not observed in COLM-2 sc tumors in nude mice (data not shown). Furthermore, we demonstrated that gefitinib markedly inhibited lung metastasis of COLM- 5 cells by more than $90 \%$ ( $\mathrm{p}<0.001$ ), and 2 of 5 mice tested became metastasis-free after 4 weeks of gefitinib treatment (Fig. 5C). We also assessed the effects of gefitinib using an experimental peritoneal dissemination model of COLM-5 cells. In control mice, peritoneal metastases progressively form peritoneal carcinomatosis with massive ascites within 2 months following injection, whereas in gefitinib-treated mice, peritoneal metastasis localized in limited areas such as the omentum and mesentery without ascites formation (Fig. 5D). Metastatic tumor weight in the peritoneal cavity was significantly decreased in gefitinib-treated mice as compared with non-treatment control $(\mathrm{p}<0.01)$.

HDAC inhibitors upregulate HER3 expression in COLM-5 cells. Next we hypothesized that HER3 deficiency of COLM-5 cells might reflect their undifferentiated phenotype. To test this, we treated COLM-5 cells with sodium butyrate (NaBT), an HDAC inhibitor, an established inducer of differentiation. NaBT treatment significantly induced expression of E-cadherin in COLM-5 cells, indicating enhancement of epithelial differentiation, although MUC2 and villin were not significantly affected (data not shown). A flow cytometric analysis demonstrated that NaBT increased HER3 expression, but not EGFR and HER2 expression on the surface of COLM-5 cells (Fig. 6A). HER3 mRNA was also increased by NaBT treatment (Fig. 6B). A western blot analysis confirmed significant upregulation of HER3, P-HER3 and P-Akt in COLM-5 cells, indicating activation of HER3/PI3K/Akt signaling by NaBT. Though gefitinib almost completely inhibited phosphorylation of HER3 and Akt in the control, it failed to suppress NaBT-mediated upregulation of P-Akt (Fig. 6C). Interestingly, p2 $7^{\mathrm{Kip} 1}$ expression was increased by gefitinib treatment with concomitant decrease in P-Akt. Such a gefitinib-mediated upregulation of p2 $7^{\mathrm{Kip} 1}$ was reduced with activation of HER3/Akt signaling by NaBT treatment (Fig. 6C), indicating the presence of a HER3/PI3K/ Akt-mediated negative regulatory mechanism of $\mathrm{p} 27^{\mathrm{Kip} 1}$ in COLM-5 cells. Treatment with NaBT at a lower concentration $(0.25 \mathrm{mM})$ for $6-12 \mathrm{~h}$ induced HER3 expression, without phenotypic change such as growth retardation (Fig. 6D). Such an upregulation of HER3 by NaBT resulted in significant restoration of gefitinib-mediated growth inhibition in COLM-5 cells $(\mathrm{p}<0.05)$ (Fig. 6E).

HER3 overexpression attenuates gefitinib-sensitivity in COLM-5 cells. In order to test whether HER3 would affect gefitinib-sensitivity, we established COLM-5 cells that stably overexpress HER3. Increased expression of more than 4-fold median intensity of HER3 protein on cellular surface was confirmed by FACS analysis and also by western blot analysis (Fig. 7A). The forced expression of HER3 induced significant resistance toward gefitinib in COLM-5 cells in vitro (Fig. 7B). Furthermore, we examined efficacy of gefitinib for COLM-5 xenografts in mice. HER3 overexpression was also confirmed by an immunohistochemical analysis of sc tumors in mice 
A

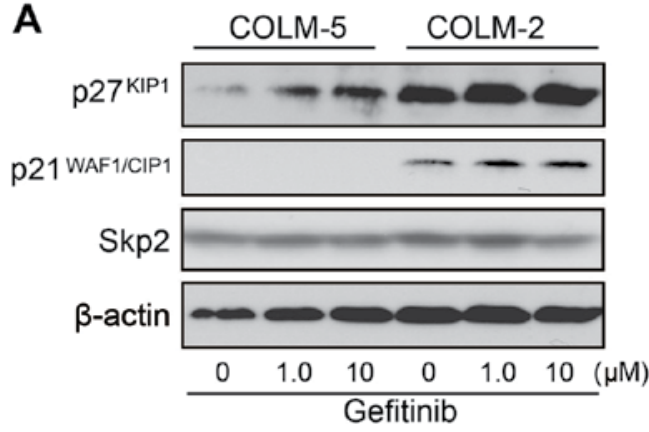

C

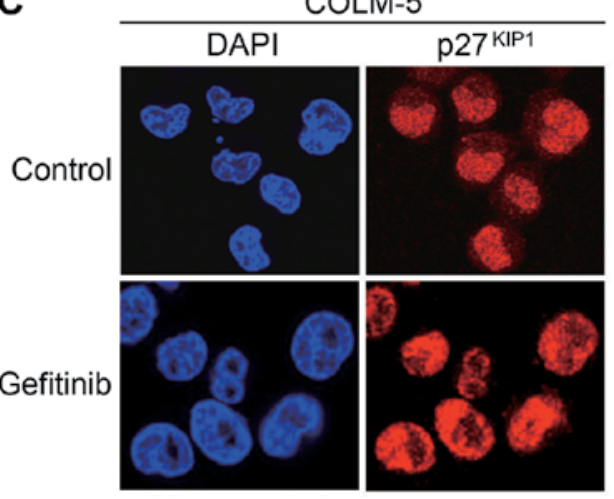

B

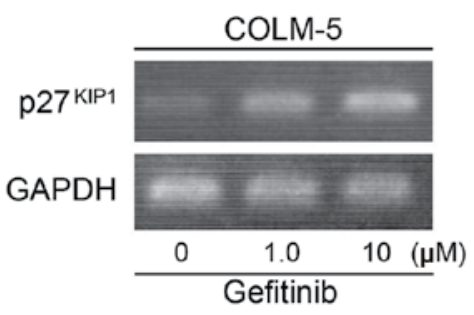

D

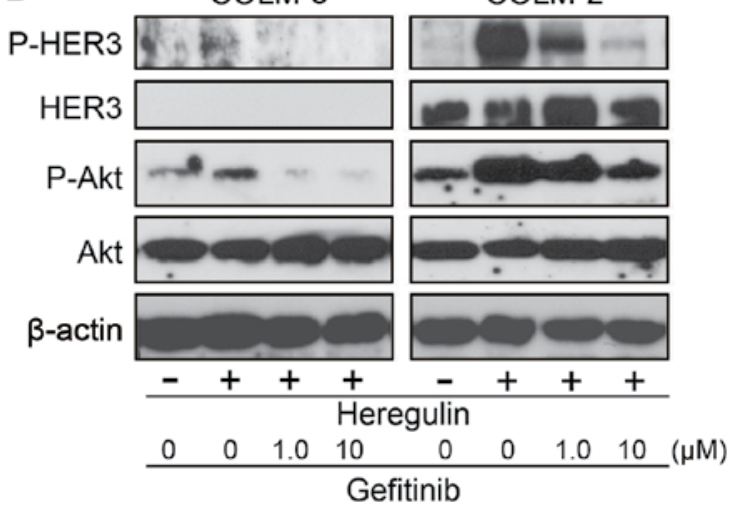

Figure 4. Effects of gefitinib on $\mathrm{p} 27^{\mathrm{Kip1}}, \mathrm{p} 21^{\mathrm{WAF} / \mathrm{CIP1}}$, Skp2 expression, and HER3/PI3K/Akt signaling. (A) Western blot analysis of p2 $7^{\mathrm{Kip1}}, \mathrm{p} 21^{\mathrm{WAF} / \mathrm{CIP1} 1}$ and Skp2 in COLM-5 and COLM-2 cells treated with gefitinib. (B) RT-PCR analysis of p27 $7^{\mathrm{Kipl}}$ in COLM-5 cells treated with gefitinib. (C) Subcellular localization of p27 $7^{\mathrm{Kipl}}$ by immunofluorescence microscopy. Both cytoplasmic and nuclear staining of $\mathrm{p} 27^{\mathrm{Kipl}}$ is observed in control cells, but only nuclear staining of p27 ${ }^{\mathrm{Kipl}}$ is seen in gefitinib-treated COLM-5 cells. (D) Western blot analysis of HER3/Akt signaling in COLM-5 and COLM-2 cells treated with gefitinib under heregulin stimulation.

A
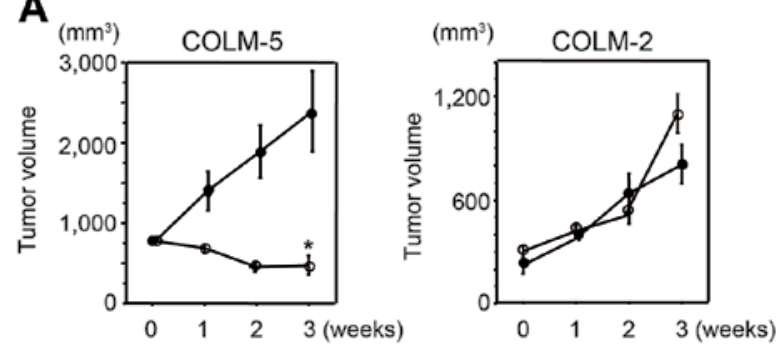

C
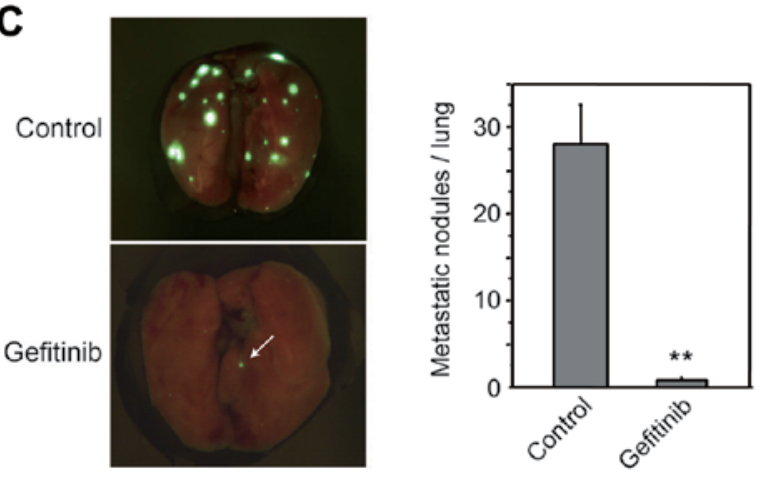

B
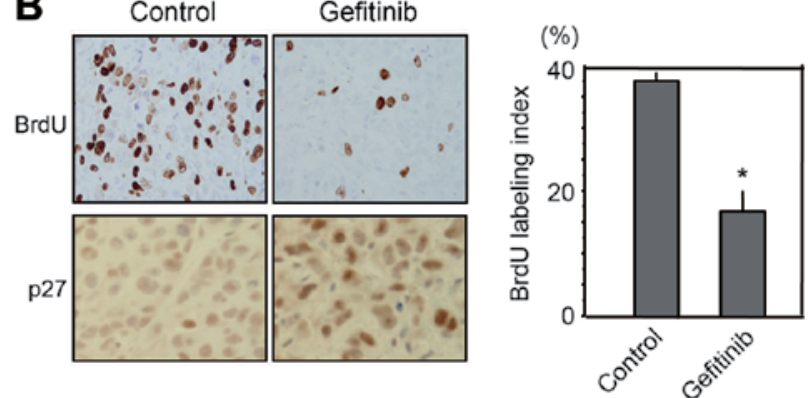

D
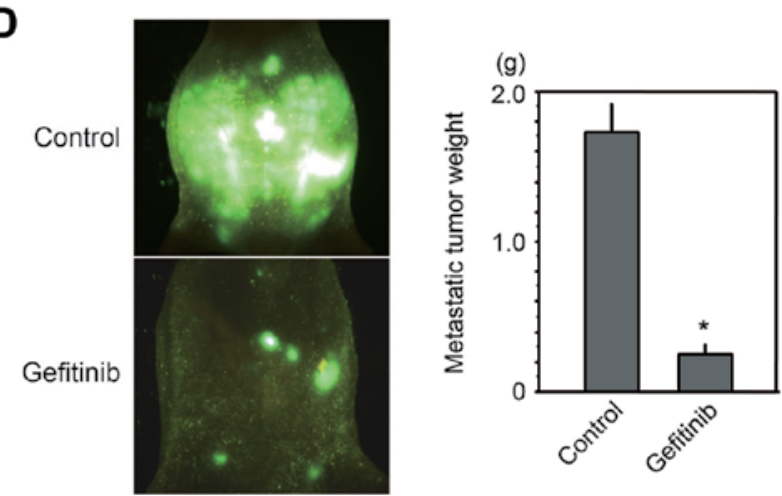

Figure 5. Effects of gefitinib on the growth and metastasis of COLM-5 cells in nude mice. (A) Growth curves of subcutaneous (sc) tumors. Vehicle control (•), gefitinib treatment (O). (B) Left panel, BrdU and p2 $7^{\mathrm{Kipl}}$ staining of gefitinib-treated and control sc tumor. Note increase in nuclear p2 $7^{\mathrm{Kipl}}$ staining in the gefitinibtreated tumor. Right panel, inhibitory effects of gefitinib on the BrdU labeling index. (C) Inhibition of experimental lung metastases of COLM-5 cells by gefitinib. Arrow indicates residual micrometastasis after treatment. (D) Inhibition of peritoneal metastases of COLM-5 cells by gefitinib. In gefitinib-treated mice, metastases are limited to the omentum and mesenterium. ${ }^{*} \mathrm{p}<0.01,{ }^{* *} \mathrm{p}<0.001$. Bars are mean $\pm \operatorname{SE}(\mathrm{n}=5)$. 
A
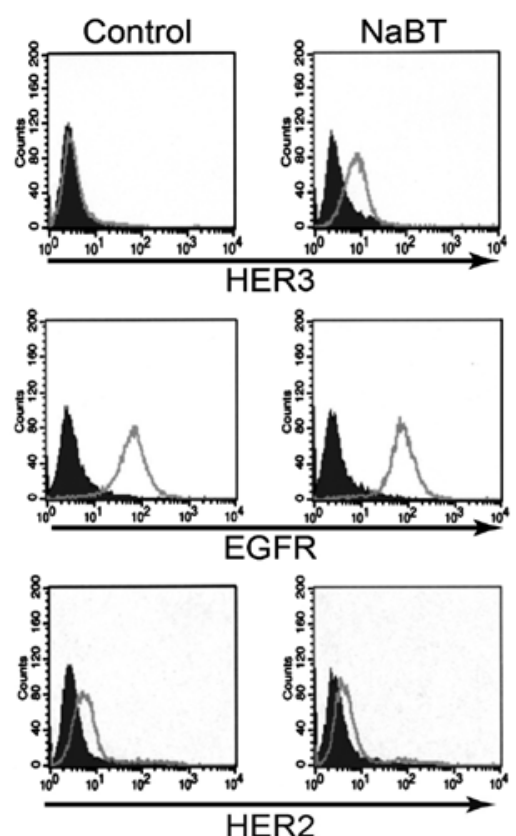

D

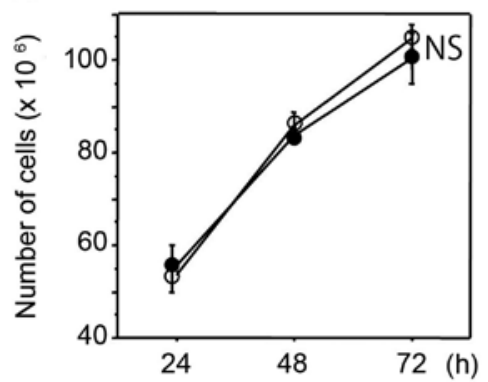

B

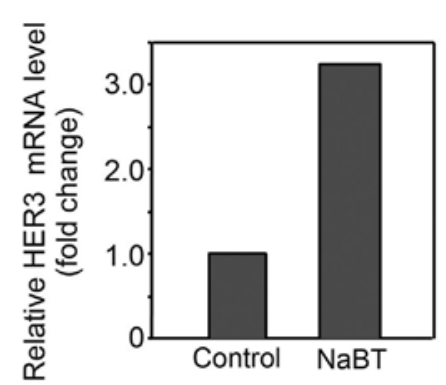

C
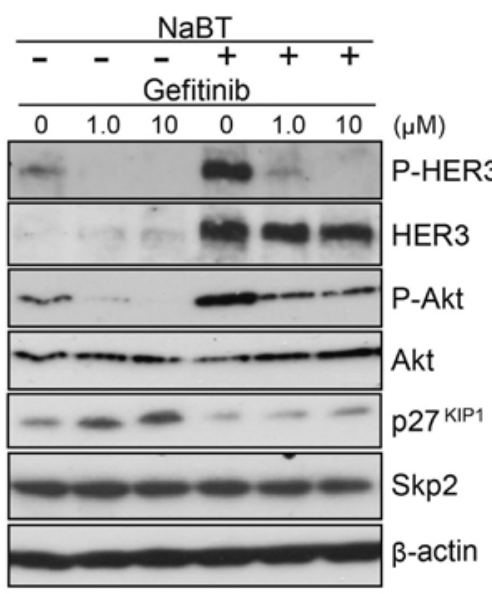

E

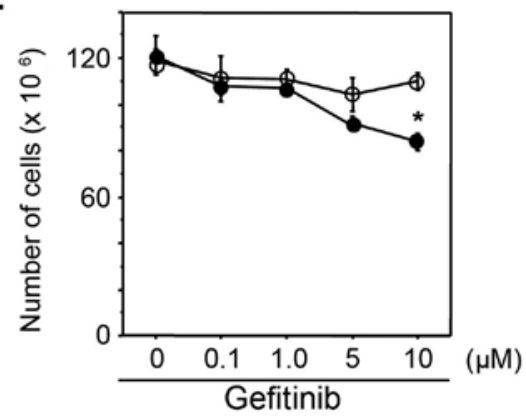

Figure 6. Sodium butyrate upregulates HER3 expression and attenuates effects of gefitinib. (A) Upregulation of HER3 by NaBT as revealed by FACS analysis. (B) Upregulation of only HER3 mRNA by NaBT as measured by quantitative RT-PCR. (C) Effects of upregulation of HER3 by NaBT on the HER3/PI3K/Akt signaling pathway and P27 ${ }^{\mathrm{Kipl}}$ expression in COLM-5 cells as revealed by western blot analysis. (D and E) Effects of upregulation of HER3 by NaBT on the gefitinib sensitivity. NaBT treatment did not significantly affect proliferation (D), but restored gefitinib-mediated growth inhibition (E). Control (•), NaBT treatment (O). * $\mathrm{p}<0.05$. NS, not significant. Bars are mean \pm SE.

(Fig. 7C). Such an increased expression of HER3 resulted in a significant induction of gefitinib-resistance of the COLM-5 sc xenografts in mice (Fig. 7D).

\section{Discussion}

In the present study, we demonstrated that the incidence of deficient HER3 expression in PD-CRC cases was much higher than WMD-CRC, indicating that HER3 expression in CRC correlates with their degree of histological differentiation. To our knowledge, there are four immunohistochemical studies that investigated HER 3 expression in CRC tissues, but only one study reported HER3 expression in PD-CRC cases, even in a limited number of PD cases $(n=8)$. They showed no HER3 expression $(0 / 8)$ in PD-CRC cases, supporting our present findings (30). Because of the relatively rare incidence of PD-CRC, our present immunohistochemical study of HER3 expression focusing on PD-CRC $(n=24)$ is, to our knowledge, the first detailed analysis to be reported in this respect. The other three studies did not report HER 3 expression of CRC according to their histological differentiation. Two studies reported the relationship between HER 3 overexpression and patient's outcome, but there is some controversy. One study showed HER3 overexpression was associated with a poor outcome of CRC patients (31), whereas two other studies including a recent meta-analysis in 12 studies showed that HER3 overexpression could not be linked with poor prognosis in CRC, despite association with short survival in patients with several types of solid tumors, including breast, ovarian cancers and melanoma $(32,33)$. The reason for this discrepancy between studies may be primarily due to the difference in the antibody used in these studies. HER3 staining pattern differed from cytoplasmic predominant staining to partially 
A

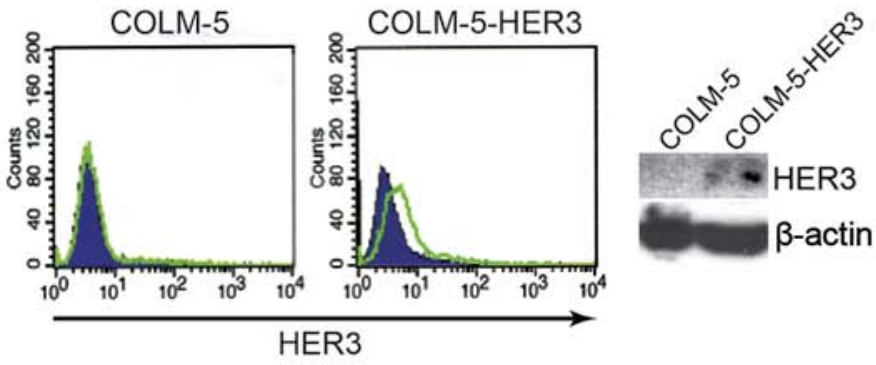

C

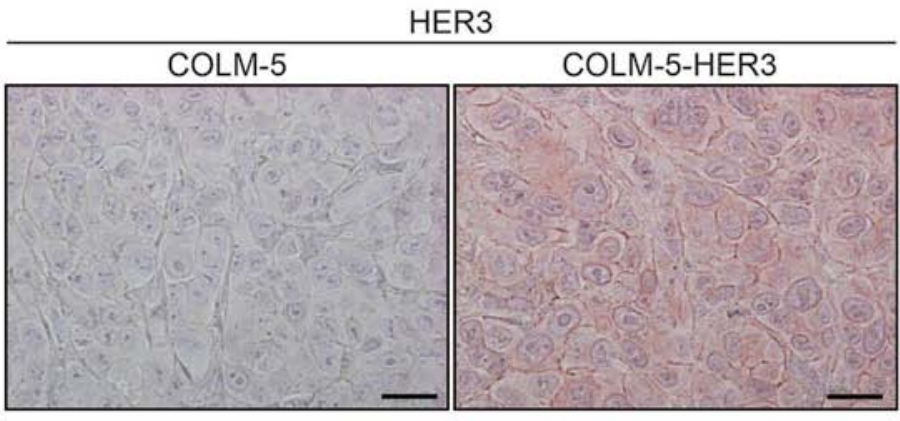

B $(\%)$

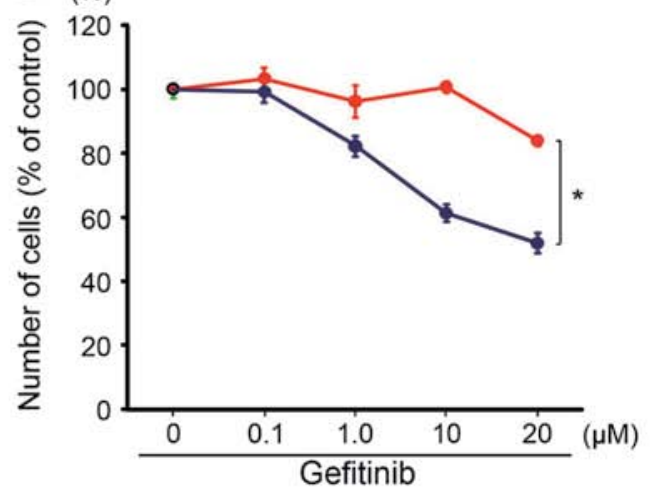

D

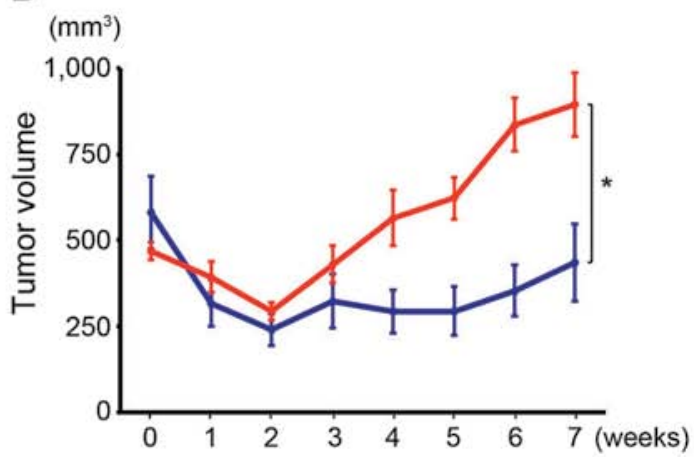

Figure 7. Forced expression of HER3 attenuates gefitinib sensitivity of COLM-5 cells in vitro and in vivo. (A) Upregulation of HER3 expression at protein level in the HER3-forced expressed COLM-5 cells (COLM-5-HER3) is confirmed by FACS analysis and western blot analysis. (B) Growth curves of COLM-5 cells with (red) or without (blue) overexpression of HER3 under gefitinib treatment in vitro are shown. (C) Immunohistochemical analysis of HER3 protein expression in sc xenografts of COLM-5-HER3 cells. (D) Effects of upregulation of HER3 on gefitinib sensitivity in vivo. Growth curves of COLM-5 xenografted tumors with (red, $\mathrm{n}=6$ ) or without (blue, $\mathrm{n}=10)$ HER3 overexpression under gefitinib treatment in vivo are shown. * $\mathrm{p}<0.05$. Bars are mean \pm SE.

membranous staining and the positivity rate varies greatly from 17 to $70 \%$ in colorectal cancers depending on the antibody used. A problem with HER3 immunohistochemistry is that the protocol has not been standardized internationally between the study groups, unlike immunohistochemical staining of EGFR and HER2. In this respect, the HER3 antibody used in our study was selected in terms of membrane staining among several commercially available antibodies and therefore, has a rationale for investigating functional aspects of HER3 expression by immunohistochemistry.

To investigate the effect of HER3 in the molecular targeting therapy against PD-CRC, we used a metastatic cell line (COLM-5) from a PD-CRC patient established in our laboratory without artificial gene transfection. To date, PD cell lines available are only a few such as RKO and SW620 cells. To our knowledge, COLM-5 is the first PD cell line showing high sensitivity to EGFR targeting drugs such as cetuximab and gefitinib. COLM-5 cells have the following unique characteristics: i) they exhibit no $\beta$-catenin nuclear accumulation, unlike the majority of $\mathrm{CRC}$, indicating their process of carcinogenesis is independent of constitutive activation of $\mathrm{Wnt} / \beta$-catenin signaling; ii) COLM-5 cells show significant HER family expression (score 2+) such as EGFR and HER2, but no expression of HER3 (score 0). iii) COLM-5 cells strongly express vimentin and Slug and show high invasive capability, but some epithelial characteristics still remain such as E-cadherin and CK20 expression in a limited cell population, indicating an
EMT-like feature. iv) COLM-5 cells have a high metastatic potential to multiple organs such as lung, peritoneal cavity and lymph nodes in xenograft models. These features are in sharp contrast to a typical differentiated-type CRC cell line (COLM-2), which shows activation of $\mathrm{Wnt} / \beta$-catenin signaling and gefitinib-resistance and is non-metastatic. Analysis of clinical samples as described above shows that a subset of PD with $\mathrm{EGFR}^{+} / \mathrm{HER} 2^{+} / \mathrm{HER} 3-$ phenotype like a COLM-5 cell line accounts for approximately $37 \%$ and is not uncommon in clinical PD cases, suggesting the COLM-5 cell line does not represent only a very rare $\mathrm{CRC}$ case, but reflects a relatively common PD-CRC subset.

COLM-5 cells showed high sensitivity to gefitinib when assessed both in vitro and in vivo. In fact, a subcutaneous tumor cannot substantially grow beyond initial tumor size by the treatment, and lung and peritoneal metastases were abolished by the early onset treatment with gefitinib. The COLM-5 cell line is also very sensitive to cetuximab, a chimeric monoclonal antibody to EGFR (data not shown). Concerning the mechanism underlying high gefitinib sensitivity of COLM-5 cells, we found that growth inhibition of COLM-5 cells by gefitinib was primarily due to the cell cycle arrest at G1 phase rather than induction of apoptosis. G1 arrest of the cell cycle is induced by $\mathrm{p} 21^{\mathrm{WAF} 1 / \mathrm{CIP} 1}$ and $\mathrm{p} 27^{\mathrm{Kip} 1}$, the members of the Cip/Kip family of cyclin-dependent kinase inhibitors, which suppress CDK2, thereby inhibiting phosphorylation of $\mathrm{Rb}$ protein (34). In head and neck squamous cancer cells, it is shown that 
gefitinib induces both $\mathrm{p} 21^{\mathrm{WAF} 1 / \mathrm{CIP} 1}$ and $\mathrm{p} 27^{\mathrm{Kip} 1}$, accompanied by G1 cell cycle arrest (35). In the present study, however, we found that cell cycle arrest by gefitinib in COLM-5 cells was mainly mediated by the upregulation of $\mathrm{p} 27^{\mathrm{Kip} 1}$, but not by $\mathrm{p} 21^{\mathrm{WAF} 1 / \mathrm{CIP} 1}$. The latter $\mathrm{p} 21^{\mathrm{WAF} / \mathrm{CIP} 1}$, a main target of P53, was not expressed at all in COLM-5 cells, possibly due to the lack of induction by the mutated P53 (36). We found a significant increase in $\mathrm{p} 27^{\mathrm{Kipl}} \mathrm{mRNA}$ expression after gefitinib treatment. Furthermore, we observed increased nuclear relocalization of $\mathrm{p} 27^{\mathrm{Kipl}}$ from the cytoplasm after gefitinib treatment, which possibly results from a decrease in phosphorylation of $\mathrm{p} 27^{\mathrm{Kipl}}$ at Thr157 by Akt. These results strongly suggest that both transcriptional upregulation and nuclear relocalization of p2 $7^{\text {Kipl }}$ through blockade of PI3K/Akt pathway are responsible for G1 cell cycle arrest and therefore gefitinib sensitivity of COLM-5 cells.

HER3 is known to be a key player regulating signal transduction upstream to PI3K/Akt pathways (22). In fact, with immunoprecipitation experiments, we confirmed enhancement of HER2/HER3 heterodimer formation, phosphorylation of HER3, and association of p85 regulatory subunit of PI3K within the dimer in response to stimulation with heregulin ligand in HER3 overexpressing COLM-2 cells, but such dimer formations were not evident in HER3-deficient COLM-5 cells (data not shown). Furthermore, we found that HER3/PI3K/Akt signaling can be modestly activated only when stimulated with ligand and could be completely suppressed by the gefitinib in COLM-5 cells. In contrast, HER3/PI3K/Akt signaling was markedly activated by the ligand stimulation and therefore was only partially inhibited by gefitinib in COLM-2 cells. Upregulation of $\mathrm{p} 27^{\mathrm{Kipl}}$ by gefitinib was found to closely correlate with such an abolishment of phosphorylation of HER3 and downstream Akt, suggesting that $\mathrm{p} 27^{\mathrm{Kip} 1}$ expression is tightly, and negatively regulated by HER3/PI3K/Akt signaling in COLM-5 cells. These results suggest that deficient HER3 expression plays an important role in the high gefitinib sensitivity of COLM-5 cells.

The upregulation of HER3, but not EGFR and HER2 in COLM-5 cells by sodium butyrate, an HDAC inhibitor, suppressed gefitinib-mediated $\mathrm{p} 27^{\mathrm{Kipl}}$ upregulation and resulted in reduced gefitinib sensitivity. These findings further support the idea that HER3-deficiency is responsible for the high gefitinib sensitivity of COLM-5 cells by enhancing gefitinib-mediated blockade of Akt phosphorylation and subsequent upregulation of $\mathrm{p} 27^{\mathrm{Kip} 1}$. In contrast, HER3 overexpression in COLM-2 cells might circumvent inhibitory activity of gefitinib by the constitutive activation of PI3K/Akt pathway, which results in impaired upregulation of $\mathrm{p} 27^{\mathrm{Kip} 1}$. Consistent with this, we confirmed that forced expression of HER3 in COLM- 5 cells by the transfection attenuated gefitinib sensitivity both in vivo and in vitro, confirming a direct regulatory effect of HER3 in gefitinib sensitivity of PD-CRC cells. These results are in line with the recently reported findings that siRNA- and antibody-mediated blockade of HER3 reduced proliferation and induced apoptosis in established colon cancer cell lines (31).

In conclusion, we showed a high incidence of deficient HER3 expression in PD-CRC cases, and by using a highly metastatic PD-CRC cell line with high sensitivity to gefitinib, we demonstrated for the first time that HER3-deficiency conferred gefitinib sensitivity by enhancing P $27^{\text {Kip }}$ upregulation and subsequent G1 cell cycle arrest through inactivation of PI3K/Akt signaling. To date, molecular targeting therapy for PD remains an essentially unresolved area in CRC. The present preclinical study together with analysis of clinical specimens cast new insight into molecular targeting therapy against a particular type of $\mathrm{CRC}$ and raised the possibility that a malignant subset of $\mathrm{PD}$ with $\mathrm{EGFR}^{+} / \mathrm{HER}^{+} / \mathrm{HER} 3^{-}$phenotype may be a promising new candidate as a therapeutic target for EGFR targeting drugs.

\section{Acknowledgements}

This study was supported in part by a grant from the Ministry of Health, Labor and Welfare, Japan and the Ministry of Education, Science, Sports, Culture and Technology.

\section{References}

1. Hamilton SR, Rubio CA, Vogelstein B, et al: Carcinoma of the colon and rectum. In: World Health Organization Classification of Tumours. Pathology and Genetics of Tumours of the Digestive System. Hamilton SR and Aaltonen LA (eds). IARC Press, Lyon, pp105-119, 2000.

2. Fearon ER and Vogelstein B: A genetic model for colorectal tumorigenesis. Cell 61: 759-767, 1990.

3. Fodde R, Smits R and Clevers H: APC, signal transduction and genetic instability in colorectal cancer. Nat Rev Cancer 1: 55-67, 2001.

4. Arai T, Takubo K, Sawabe M and Esaki Y: Pathologic characteristics of colorectal cancer in the elderly: a retrospective study of 947 surgical cases. J Clin Gastroenterol 31: 67-72, 2000 .

5. Kazama Y, Watanabe T, Kanazawa T, Tanaka J, Tanaka T and Nagawa H: Poorly differentiated colorectal adenocarcinomas show higher rates of microsatellite instability and promoter methylation of p16 and hMLH1: a study matched for T classification and tumor location. J Surg Oncol 97: 278-283, 2008.

6. Xiao H, Yoon YS, Hong SM, Roh SA, Cho DH, Yu CS and Kim JC: Poorly differentiated colorectal cancers: correlation of microsatellite instability with clinicopathologic features and survival. Am J Clin Pathol 140: 341-347, 2013.

7. Hinoi T, Tani M, Lucas PC, et al: Loss of CDX2 expression and microsatellite instability are prominent features of large cell minimally differentiated carcinomas of the colon. Am J Pathol 159: 2239-2248, 2001.

8. Chung CK, Zaino RJ and Stryker JA: Colorectal carcinoma: evaluation of histologic grade and factors influencing prognosis. J Surg Oncol 21: 143-148, 1982.

9. Kawabata Y, Tomita N, Monden T, et al: Molecular characteristics of poorly differentiated adenocarcinoma and signet-ring-cell carcinoma of colorectum. Int J Cancer 84: 33-38, 1999.

10. Kim JH, Rhee YY, Bae JM, Cho NY and Kang GH: Loss of CDX2/CK20 expression is associated with poorly differentiated carcinoma, the $\mathrm{CpG}$ island methylator phenotype, and adverse prognosis in microsatellite-unstable colorectal cancer. Am J Surg Pathol 37: 1532-1541, 2013.

11. Seshimo I, Yamamoto H, Mishima H, et al: Expression and mutation of SMAD4 in poorly differentiated carcinoma and signet-ring cell carcinoma of the colorectum. J Exp Clin Cancer Res 25: 433-442, 2006.

12. Rad R, Cadiñanos J, Rad L, et al: A genetic progression model of Braf (V600E)-induced intestinal tumorigenesis reveals targets for therapeutic intervention. Cancer Cell 24: 15-29, 2013.

13. Coffee EM, Faber AC, Roper J, et al: Concomitant BRAF and $\mathrm{PI} 3 \mathrm{~K} / \mathrm{mTOR}$ blockade is required for effective treatment of BRAF (V600E) colorectal cancer. Clin Cancer Res 19: 2688-2698, 2013.

14. Hynes NE and Lane HA: ERBB receptors and cancer: the complexity of targeted inhibitors. Nat Rev Cancer 5: 341-354, 2005. 
15. Oshima Y, Tanaka H, Murakami H, Ito Y, Furuya T, Kondo E, Kodera $\mathrm{Y}$ and Nakanishi $\mathrm{H}$ : Lapatinib sensitivity of two novel trastuzumab-resistant HER2 gene-amplified gastric cancer cell lines. Gastric Cancer 17: 450-462, 2014.

16. Mayer A, Takimoto M, Fritz E, Schellander G, Kofler K and Ludwig $\mathrm{H}$ : The prognostic significance of proliferating cell nuclear antigen, epidermal growth factor receptor, and mdr gene expression in colorectal cancer. Cancer 71: 2454-2460, 1993.

17. Cunningham D, Humblet Y, Siena S, et al: Cetuximab monotherapy and cetuximab plus irinotecan in irinotecan-refractory metastatic colorectal cancer. N Engl J Med 351: 337-345, 2004

18. Chung KY, Shia J, Kemeny NE, et al: Cetuximab shows activity in colorectal cancer patients with tumors that do not express the epidermal growth factor receptor by immunohistochemistry. J Clin Oncol 23: 1803-1810, 2005.

19. Moroni $\mathrm{M}$ and Veronese S: Gene copy number for epidermal growth factor receptor (EGFR) and clinical response to anti-EGFR treatment in colorectal cancer. A cohort study. Lancet Oncol 6: 279-286, 2005.

20. Lievre A, Bachet JB, Le Corre D, et al: KRAS mutation is predictive of response to cetuximab therapy in colorectal cancer. Cancer Res 66: 3992-3995, 2006.

21. Yokota T, Ura T, Shibata N, et al: BRAF mutation is a powerful prognostic factor in advanced and recurrent colorectal cancer. Br J Cancer 104: 856-862, 2011.

22. Menendez JA and Lupu R: Transphosphorylation of kinasedead HER 3 and breast cancer progression: a new standpoint or an old concept revisited? Breast Cancer Res 9: 111, 2007.

23. Sergina NV, Rausch M, Wang D, Blair J, Hann B, Shokat KM and Moasser MM: Escape from HER family tyrosine kinase inhibitor therapy by the kinase inactive HER3. Nature 445: 437-441, 2007.

24. Yakes FM, Chinratanalab W, Ritter CA, King W, Seelig S and Arteaga CL: Herceptin-induced inhibition of phosphatidylinositol-3 kinase and Akt is required for antibody-mediated effects on p27, cyclin D1, and antitumor action. Cancer Res 62 : 4132-4141, 2002.

25. Maurer CA, Friess H, Kretschmann B, et al: Increased expression of erbB3 in colorectal cancer is associated with concomitant increase in the level of erbB2. Hum Pathol 29: $771-777,1998$
26. Ito Y, Nakanishi H, Kodera Y, Hirai T, Nakao A and Kato T: Characterization of a novel lymph node metastasis model from human colonic cancer and its preclinical use for comparison of anti-metastatic efficacy between oral S-1 and UFT/ LV. Cancer Sci 101: 1853-1860, 2010 .

27. Yokoyama H, Nakanishi H, Kodera Y, et al: Biological significance of isolated tumor cells and micrometastasis in lymph nodes evaluated using a green fluorescent protein (GFP)-tagged human gastric cancer cell line. Clin Cancer Res 12: 361-368, 2006.

28. Carrano AC, Eytan E, Hershko A and Pagano M: Skp2 is required for ubiquitin-mediated degradation of the CDK inhibitor P27. Nat Cell Biol 3: 193-199, 1999.

29. Pagano M: Control of DNA synthesis and mitosis by the Skp2-p27-Cdk1/2 axis. Mol Cell 14: 414-416, 2004.

30. Kountourakis P, Pavlakis K, Psyrri A, et al: Prognostic significance of HER3 and HER4 protein expression in colorectal adenocarcinomas. BMC Cancer 6: 46, 2006.

31. Beji A, Horst D, Engel J, et al: Toward the prognostic significance and therapeutic potential of HER 3 receptor tyrosine kinase in human colon cancer. Clin Cancer Res 18: 956-968, 2012.

32. Ocana A, Vera-Badillo F, Seruga B, Templeton A, Pandiella A and Amir E: HER3 overexpression and survival in solid tumors: A meta-analysis. J Natl Cancer Inst 105: 266-273, 2013.

33. Baiocchi G, Lopes A, Coudry RA, et al: ErbB family immunohistochemical expression in colorectal cancer patients with higher risk of recurrence after radical surgery. Int J Colorectal Dis 24: 1059-1068, 2009.

34. Coqueret O: New roles for p21 and p27 cell-cycle inhibitors: a function for each cell compartment? Trends Cell Biol 13: 65-70, 2003.

35. Di Gennaro E, Barbarino M, Bruzzese F, et al: Critical role of both $\mathrm{p} 27^{\mathrm{KIP} 1}$ and $\mathrm{p} 21^{\mathrm{CIP} 1 / \mathrm{WAF} 1}$ in the antiproliferative effect of ZD1839 ('Iressa'), an epidermal growth factor receptor tyrosine kinase inhibitor, in head and neck squamous carcinoma cells. J Cell Physiol 195: 139-150, 2003.

36. Varna M, Lehmann-Che J, Turpin E, et al: p53 dependent cell-cycle arrest triggered by chemotherapy in xenografted breast tumors. Int J Cancer 124: 991-997, 2009. 